\title{
Neurobiología del juego patológico: manifestaciones clínicas e implicaciones neuropsicológicas ${ }^{1}$
}

Neurobiology of pathological gambling: clinical manifestations and neuropsychological implications

Neurobiologia do jogo patológico: manifestações clínicas e implicações neuropsicológicas

\section{Jorge Alexander Ríos-Flórez² \\ Carolina Escudero-Corrales ${ }^{3}$}

Recibido: 10.10.2016 • Arbitrado:08. 11.2016• Aprobado: 19.11.2016

\section{Resumen}

Pese a categorizarse actualmente como un trastorno de adicción, el ‘juego patológico’ no se relaciona exclusivamente con el uso y dependencia de sustancia químicas, aunque sus efectos y manifestaciones

${ }^{1}$ Artículo producto del macroproyecto "Alteraciones Neurofuncionales en los Trastornos mentales", formulado en la línea de Investigación Psicopatología y Neuropsiquiatría, vinculada al Grupo de Estudio e Investigación en Neurociencias Hippocampus. Fecha de inicio del subproyecto Septiembre de 2016, Fecha de finalización Noviembre de 2016.

${ }^{2}$ Investigador, Director del Grupo de Estudio e Investigación en Neurociencias HIPPOCAMPUS, Colombia, Especialista en Evaluación y Diagnóstico Neuropsicológico, Magíster en Neuropsicología Clínica, Universidad de San Buenaventura Bogotá, D.C. Facultad de Ciencias, Sociales Institución Universitaria Politécnico Grancolombiano; Docente de la Facultad de Educación y Ciencias Sociales, Tecnológico de Antioquia Institución Universitaria, Medellín, Colombia, alexanderriosflorez@gmail.com, jriosflo@poligran.edu.co.

${ }^{3}$ Investigadora, Miembro del Grupo de Estudio e Investigación en Neurociencias Hippocampus, Colombia. Psicóloga, Universidad Pontificia Bolivariana, Medellín, Colombia, carolina.escudero@upb.edu.co. 
emocionales, cognitivas y conductuales son claramente similares, de allí a que se le considere como el único trastorno de adicción sin drogas clasificado en el DSM-5. A nivel clínico, es importante considerar variables sociodemográficas, tipología, criterios diagnósticos, posible patología dual y presencia de alteraciones cognitivas, conductuales y neuropsicológicas que permitan realizar un diagnóstico diferencial adecuado y con ello establecer propuestas de evaluación e intervención eficaces. Respecto a las afecciones neuropsicológicas, se destacan implicaciones importantes en la toma de decisiones, control emocional, inhibición e iniciativa comportamental, siendo todos estos componentes claves de las funciones ejecutivas. Así mismo, se ha reportado que el déficit de la función ejecutiva en el jugador patológico es similar al evidenciado en pacientes diagnosticados con trastorno de adicción por consumo de sustancias psicoactivas e incluso activación similar de los sistemas de recompensa neuronales.

Palabras clave: Adicción, Cognición, Juego patológico, Lóbulos frontales, Sistema de recompensa.

\section{Abstract}

Even though pathological gambling is categorized as an addictive disorder, it is the only one not related to drugs considered by the DSM5 , since it causes similar consequences at the emotional, cognitive and behavioral level such as any other addictive disorders related to the use and dependence of chemical substances. At the clinical level, it is important to consider sociodemographic variables, typology, diagnostic criteria, possible dual pathology and the presence of cognitive, behavioral and neuropsychological alterations that allow an adequate differential diagnosis and the set of effective evaluation and intervention proposals. Regarding neuropsychological conditions, affected decision making, emotional control, inhibition and behavioral activation, stand out, all of them key components of executive functions. Likewise, it has been reported that the deficit of the executive functioning in the pathological player is like the one evidenced in patients with diagnosis of addiction disorder due to the use of psychoactive substances and even similar activation of the neural reward systems. 
Keywords: Addiction, Cognition, Frontal lobes, Neuropsychology, Pathological gambling, Reward system.

\section{Resumo}

O jogo patológico além de ser atualmente classificado como um transtorno do comportamento aditivo, não está relacionado exclusivamente com o uso e dependência de substância química. Porem, os seus efeitos e manifestações emocionais, cognitivas e comportamentais tornassem fortemente similares. É por isto, que seja considerado como a única desordem de tipo aditivo que é possível de diagnosticar sem o uso de drogas proposto na DSM-5. A nível clínico é importante considerar variáveis de ordem sociodemográficas, tipologia, critérios diagnósticos, possível dupla patológica e presença de alterações cognitivas, comportamentais e neuropsicológicas que permitam fazer o diagnóstico diferencial adequado e, assim, estabelecer propostas de avaliação e intervenção efetivas. O quem tem a ver com os déficits neuropsicológicos, são bem conhecidos os prejuízos especialmente na tomada de decisões, o controle emocional, inibição e a iniciativa comportamental, componentes chaves das funções executivas. Adicionalmente, tem sido relatado que o déficit na função executiva no jogador patológico é similar aos sintomas avaliados nos pacientes como o diagnostico de dependência por abuso de substâncias psicoativas, incluso, uma ativação semelhante dos sistemas de recompensa a nível neural.

Palavras chave: Addiction, Cognição, Jogo patológico, Lobos frontais, Sistema de recompensa. 


\section{Introducción}

Pese a ser el juego de azar una tradición antiquísima presente en gran parte de las culturas, solo hasta hace algunos años se constituyó como un problema de salud pública, en tanto que ha ascendido considerablemente la prevalencia de jugadores, particularmente de adolescentes y adultos jóvenes debido, primordialmente, a la legalización de establecimientos destinados a este entretenimiento, como casinos, bingos, bares, entre otros, lo que implica un aumento desmedido de estos (Ochoa et al., 1994; Unwin, Davis \& De Leeuw, 2000). Lo anterior, se ha relacionado, a su vez, con mayores tasas de criminalidad, violencia y drogadicción, particularmente en la población adolescente (Unwin et al., 2000). Cada vez se asocian a esta práctica, en niveles patológicos, alteraciones relacionadas con el comportamiento, los productos psicológicos y de funcionalidad neurológica.

Aunque la prevalencia es menor en comparación con países europeos como España, en donde el juego se constituye como una de las principales fuentes de ingreso económico (Comisión Nacional del Juego, 2001), en Colombia aproximadamente el 61\% de la población invierte gran parte de sus recursos mensuales en juegos de azar y la prevalencia entre clases sociales es comparativamente similar; además, este porcentaje ha ido en aumento desde su legalización (Bahamon, 2006).

No obstante, otros estudios plantean que no existen cifras contundentes respecto al juego patológico en población Colombiana, por lo que se considera a este trastorno como una problemática de alto impacto y poco control nacional, ya que han sido escasos los esfuerzos por conocerla, diagnosticarla y tratarla oportunamente, en comparación a otros países que se han dirigido a realizar diversos estudios desde el campo de la psicología y la neuropsicología (Bahamon, 2006) en aras de comprender su etiología, tipología, comorbilidad y principales afecciones emocionales cognitivas y conductuales.

A propósito del campo de la neuropsicología, son también escasos los estudios realizados en Colombia y en la actualidad no se constatan en la literatura posibles propuestas de rehabilitación de las funciones mentales más afectadas con este trastorno. 


\section{Conceptualización y generalidades}

En 2014 el Manual Diagnóstico y Estadístico de los trastornos mentales en su quinta y más reciente edición (DSM-5) realizó una serie de modificaciones respecto a la clasificación de algunos trastornos, entre ellos el juego patológico, que en la versión anterior (DSM-IV) se hallaba dentro de la categoría de 'Trastornos del control del impulso' y ahora se reubica en la categoría de 'Trastornos relacionados con sustancias y trastornos adictivos', reconociéndose dentro de esta clasificación como un trastorno adictivo no relacionado con sustancias, pero con características etiopatogénicas, emocionales, cognitivas y conductuales, similares a los trastornos adictivos por consumo de sustancias, en tanto que activan de forma semejante los mismos sistemas de recompensa que se ponen en acción en pacientes drogadictos. Actualmente, el juego patológico se ha considerado el único trastorno de adicción no relacionado con sustancias que se ha incluido dentro de la categoría mencionada (American Psychiatric Association [APA], 2014).

Con respecto a la prevalencia de este trastorno, se considera que su aparición oscila entre un 0.2 y $0.3 \%$ respecto a la población general. De igual forma, se constata que la prevalencia en el género femenino es de $0.2 \%$ aproximadamente y en el género masculino es del $0.6 \%$. Asimismo, se cree que el inicio de este trastorno puede evidenciarse tanto en la adolescencia como en la adultez temprana y va progresando de forma rápida e insidiosa con el transcurrir de los años, hasta tal punto de ser incontrolable y recurrente, causando malestar o displacer significativo en el sujeto. Es importante resaltar que el tipo de juego de azar, la cantidad, frecuencia, intensidad, incidencia, patrones, montos de inversión e influencias sociales y ambientales, no constituyen factores exclusivos o determinantes para diagnosticar juego patológico, ya que estas variables tienden a variar entre persona y persona; y en razón de ello es necesario contemplar los criterios diagnósticos requeridos para su diagnóstico (APA, 2014).

Las manifestaciones iniciales del trastorno suelen ser más frecuentes en la adultez joven y específicamente en hombres, quienes 
acuden escasamente a tratamiento, en comparación con las mujeres que suelen presentar el trastorno en etapas más tardías y recurren con mayor facilidad a tratamiento, aunque de manera general, el porcentaje de personas con esta patología que recurren a ayuda farmacológica y psicológica es irrisoria (APA, 2014).

Fisiopatológicamente, el juego patológico comparte ciertas manifestaciones cognitivas y conductuales semejantes a otros trastornos de adicción por sustancias, como es el caso del abuso de alcohol. Esta relación se ha expuesto a través del paradigma del sesgo atencional por medio del cual se explica cómo el jugador comienza a presentar gran atención y motivación hacia estímulos especificos relacionados con el juego, determinándose una atención selectiva frente a los mismos que se mantienen en el tiempo, lo que dificulta desprenderse o desengancharse del juego, así como atender a otro tipo de estímulos diferentes. Este sesgo atencional incrementa, a su vez, los niveles de deseo, gratificación e impulsividad (Verdura, 2014).

\section{Tipología del cuadro patológico}

Ya se mencionó con anterioridad que la característica principal del jugador patológico es la manifestación de un comportamiento recurrente y persistente respecto al juego, que afecta considerablemente las dimensiones de su vida en general; no obstante, para considerar el nivel de gravedad o afectación es necesario puntualizar o especificar en qué nivel se encuentra o a qué tipo de jugador se hace referencia. Con respecto a esto, el DSM-5 no enfatiza ni aborda una tipología particular, pero hace especial énfasis en posibles especificadores que miden el nivel de gravedad en cada caso, respecto a los criterios diagnósticos establecidos en el trastorno. De este modo, el DSM-5 considera importante especificar si el trastorno es de tipo episódico o persistente, si presenta periodos de remisión parcial que oscilan entre los 3 a los 12 meses máximo, o si presenta periodos de remisión continuada que se extienden posterior a los 12 meses. Así mismo, se debe especificar de acuerdo a la gravedad actual del sujeto en relación a la cantidad de criterios que este cumple. En este sentido, el jugador patológico leve 
es aquel que cumple con 4 o 5 de los 9 criterios establecidos y que se caracteriza por manifestar cierto grado de malestar o preocupación ante las pérdidas monetarias. Por su parte, el jugador patológico moderado, cumple 6 o 7 de los 9 criterios establecidos, mientras que el jugador patológico grave cumple todos o casi todos los criterios establecidos, presentando mayores implicaciones y pérdidas en las diferentes esferas de su vida cotidiana (APA, 2014).

Otra clasificación atinente a este trastorno es presentada por autores como Blaszczynski \& Nover (2002) quienes abordan al jugador patológico en tres tipos, siendo el tipo I el jugador puro,, el cual presenta a grandes rasgos toda la sintomatología observable del típico jugador patológico; el tipo II el jugador con alta vulnerabilidad emocional,, que presenta mayor predisposición biológica y ambiental a presentar conductas incontrolables e impulsivas ante el juego y a manifestar rasgos de personalidad propios de esta población; y el tipo III o jugador multiimpulsivo con otras patologías asociadas, caracterizado por una alta dificultad para abandonar el juego y presencia de afecciones conductuales, cognitivas y emocionales que se encuentran relacionadas a su vez con la presencia de otros trastornos comórbidos (Blaszczynski \& Nover, 2002).

A esta categorización se ha incluido un cuarto tipo denominado enfermo mental que juega, el cual presenta diagnóstico de juego patológico y a la par algún trastorno mental considerablemente grave como los trastornos del espectro de la esquizofrenia y otros trastornos psicóticos, o trastorno bipolar (González et al., 2003; Echeburúa, 2006; Gómez, 2006; Gómez, 2015). Por su parte, otros autores como Prieto (2003), han sugerido una clasificación diferente, refiriéndose a los tipos de jugadores buscadores de activación, jugadores buscadores de dinero y jugadores de escape.

Las anteriores clasificaciones hacen alusión a tipos o niveles de manifestación en el juego patológico de manera particular, sin embargo, se han realizado otras clasificaciones más amplias, que incluyen al jugador patológico y a otros tipos de jugadores que pue- 
den llegar a confundirse al momento de realizar un diagnóstico de juego patológico. Asi pues, se incluye dentro de esta categorización al jugador social, jugador profesional, jugador problema y jugador patológico (Ochoa et al., 1994).

Dentro de las características principales del jugador social se destaca el jugar como una forma de entretenimiento y placer que realiza con grupos de amigos por periodos de tiempos delimitados y en espacios de ocio. Este tipo de jugador planifica y es consciente de cuándo y cuánto invertir, sin que sea algo necesario y recurrente que implique problemas personales, familiares o laborales. Juega cuando lo desea sin ser esta la actividad que ocupa la mayor parte de su tiempo y concibe el juego como una actividad temporal para compartir y divertirse socialmente (Ochoa et al.,1994; Ochoa et al., 2013; APA, 2014).

Por su parte, el jugador profesional se reconoce por ser un sujeto estratégico, metódico y calculador; implementa técnicas estadísticas y/o de probabilidad al momento de apostar, por lo que juega exclusivamente en ciertos espacios y situaciones en los que sabe que tiene más posibilidades de ganar. Es usual ver a este tipo de jugadores en lugares como casinos (Ochoa et al.,1994).

De otro lado, el jugador problema presenta un menor control de la conducta de juego, que le conlleva a invertir desmesuradamente altas cantidades de dinero u objetos de valor ajenos como bienes familiares. No obstante, dicha dificultad para controlarse es menor que en el caso del jugador patológico, pero mayor si se le compara con el jugador social. En general, este tipo de jugadores presenta mayor tendencia o predisposición a convertirse en un jugador patológico (Ochoa et al.,1994; Ochoa et al., 2013).

En última instancia se encuentra el jugador patológico que, tal como se ha puntualizado en apartados previos, se caracteriza por una imposibilidad para controlar sus conductas en el juego y que conlleva a un malestar significativo y deterioro en las diferentes áreas en las que se desenvuelve el sujeto (Ochoa et al.,1994; APA, 2014). 


\section{Factores etiológicos}

De manera general, se contemplan predisposiciones a nivel genético, temperamental/ambiental. En el primer caso se considera que el juego patológico es más común en gemelos monocigóticos y en familiares de personas con trastornos por consumo de sustancias, específicamente por abuso de alcohol (APA, 2014). A nivel ambiental se consideran inicios tempranos en juegos de azar, particularmente en la infancia y la adolescencia (Lesieur \& Rosenthal, 1991; American Psychiatric Association [APA], 2014) y comorbilidad con trastornos de personalidad como el trastorno antisocial, así como trastornos bipolares y depresivos.

Otros factores de riesgos asociados a la patología son la legalización de los juegos de azar que incrementa la prevalencia del trastorno, especialmente en la adolescencia y la adultez joven (Ochoa et al., 1994), situación laboral precaria o desempleo (Volberg \& Abbott, 1994) y la influencia de padres jugadores o alcohólicos (Zeitlin, 1994; Butcha, 1995).

\section{Criterios diagnósticos}

En abordaje clínico del trastorno se toman como punto de partida los criterios diagnósticos presentados en el Manual Diagnóstico y Estadístico de los trastornos mentales, quinta edición (DSM-5) y la Clasificación internacional de enfermedades, décima versión (CIE-10), siendo el primero un sistema de clasificación más amplio y descriptivo, mientras que el segundo presenta de manera general los principales criterios. 
Tabla 1. Criterios diagnósticos del juego patológico según DSM-5

\section{Criterios diagnósticos del juego patológico según DSM-5}

A. Juego patológico problemático persistente y recurrente que provoca un deterioro o malestar clínicamente significativo y se manifiesta porque el individuo presenta cuatro (o más) de los siguientes criterios durante un periodo de 12 meses:

1. Necesidad de apostar cantidades de dinero cada vez mayores para conseguir la excitación deseada.

2. Está nervioso o irritado cuando intenta reducir o abandonar el juego.

3. Ha hecho esfuerzos repetidos para controlar, reducir o abandonar el juego, siempre sin éxito.

4. A menudo tiene la mente ocupada en las apuestas (p. ej., reviviendo continuamente con la imaginación experiencias de apuestas pasadas, condicionando o planificando su próxima apuesta, pensando en formas de conseguir dinero para apostar).

5. A menudo apuesta cuando siente desasosiego (p. ej., desamparo, culpabilidad, ansiedad, depresión).

6. Después de perder dinero en las apuestas, suele volver otro día para intentar ganar ("recuperar" las pérdidas).

7. Miente para ocultar su grado de implicación en el juego.

8. Ha puesto en peligro o ha perdido una relación importante, un empleo o una carrera académica o profesional a causa del juego.

9. Cuenta con los demás para que le den dinero para aliviar su situación financiera desesperada provocada por el juego.

B. Su comportamiento ante el juego no se explica mejor por un episodio maniaco.

Fuente: Tomado de DSM-5. American Psychiatric Association (2014).

Comparativamente se encuentran diferencias significativas entre el DSM-5 y el CIE-10; así pues, el DSM-5 define al juego patológico como un trastorno persistente y recurrente que causa malestar o displacer significativo en el individuo así como deterioro en las dimensiones laboral, educativa, familiar y social y cuyas conductas particulares se presentan durante al menos 1 año, cumpliendo 4 o más 
de los 9 criterios o ítems establecidos para su diagnóstico y descartando la presencia de posibles estados maniacos (Tabla 1). Por el contrario en el CIE-10 se encuentran descritos solo 4 criterios o ítems, que deben cumplirse en su totalidad y no plantea criterios de exclusión como en el caso del DSM-V en el que se descartan los episodios maníacos para explicar la conducta observada (Tabla 2).

Tabla 2. Criterios diagnósticos del juego patológico según CIE-10

Criterios diagnósticos del juego patológico según CIE-10
A. Presencia de dos o más episodios en un periodo de al menos un año.
B. Estos episodios carecen de provecho económico para el individuo y, sin embargo, se reiteran a pesar de los efectos perjudiciales que tienen a nivel social y laboral, y sobre los valores y compromisos personales.
C. El sujeto describe la presencia de un impulso intenso de jugar difícil de controlar,
y afirma ser incapaz de dejar de jugar mediante el único esfuerzo de su voluntad.
D. Preocupación con pensamiento o imágenes mentales relacionados con el acto de jugar o con las circunstancias que lo rodean.

Fuente: Tomado de CIE-10. Organización Mundial de la Salud (1994).

\section{Comorbilidades y diagnóstico diferencial}

Cuando se considera un trastorno mental, en este caso el juego patológico, es importante contemplar posibles trastornos comórbidos; al respecto, el DSM-5 plantea que personas diagnosticadas con juego patológico pueden presentar, a la par, trastornos por consumo de sustancias, trastornos de bipolaridad, trastornos depresivos, trastornos de ansiedad y ciertos trastornos de personalidad. El reconocer la comorbilidad con otros trastornos permite realizar un diagnóstico más veraz que conduzca a un tratamiento de mayor eficacia (APA, 2014).

De otro lado, al momento de realizar un diagnóstico diferencial se hace ineludible excluir o descartar algunos trastornos con los que el juego patológico se puede confundir a nivel sintomatológico, o de criterios clínicos, entre ellos, el trastorno de personalidad antisocial y epi- 
sodios maníacos, asi como manifestaciones clínicas similares por uso de algun medicamento o por una afección médica particular. De igual forma, se debe distinguir claramente el juego patológico del juego social y profesional que no representan riesgos considerables ni tampoco cumplen con los criterios diagnósticos establecidos (APA, 2014).

\section{Implicaciones neuropsicológicas del juego patológico}

En la actualidad son escasas las investigaciones relacionadas con alteraciones neuropsicológicas en jugadores patológicos, no obstante, aquellas reportadas en la literatura se han centrado en evaluar ciertas dimensiones de la función ejecutiva, como inhibición, iniciativa, organización, planeación, control emocional, monitoreo y flexibilidad, encontrando diferencias significativas en estos componentes en comparación con el grupo control (Zuluaga \& Montoya, 2005).

En la evaluación de cada una de las dimensiones mencionadas, $\mathrm{Zu}-$ luaga y Montoya, (2005), reportaron de manera general en el grupo clínico, un alto nivel de impulsividad, volubilidad, falta de control emocional, irritabilidad, conductas explosivas o reactivas, baja tolerancia a la frustración, dificultades en la resolución de problemas, implicaciones en la atención sostenida y selectiva, poco interés en las relaciones sociales, poca motivación y creatividad frente a ciertas situaciones o tareas, baja autoconciencia, poca responsabilidad y autocontrol respecto a su trastorno y a sus actos, además de incapacidad para planear, establecer y anticipar metas o sucesos futuros.

Así mismo, es importante resaltar que el control, la inhibición y la iniciativa son las dimensiones más implicadas durante las sesiones de juego Dichas alteraciones, suelen estar acompañadas de sesgos respecto a la probabilidad o riesgo, y déficits a nivel de la memoria selectiva (Monaghan, Blaszczynski \& Nower, 2009). Se han evidenciado además, alteraciones en la memoria de trabajo respecto al funcionamiento ejecutivo (Forbush et al., 2008; Marazziti et al., 2008), encontrándose una relación entre errores por perseveración y déficit en el funcionamiento de la corteza prefrontal dorsolateral; lo que explica una disminución en la flexibilidad 
cognitiva (Leiserson \& Pihl, 2007). Aunque en general, no se ha llegado a un consenso, pues otros estudios (Cavedini et al., 2002) reportan no hallar ninguna alteración en componentes de flexibilidad mental.

También se han realizado correlaciones entre jugadores patológicos (con conductas adictivas no relacionadas con sustancias) y pacientes con conductas adictivas por sustancias y se ha encontrado, en ambos, alteraciones similares a nivel atencional y déficit en el componente de toma de decisiones de la función ejecutiva asociado a inflexibilidad cognitiva (Rugle \& Melamed, 1993; Barry \& Petry, 2008), especialmente frente a tareas que implican probabilidad, siendo más notoria esta dificultad ante situaciones que suponen recompensas futuras con poco riesgo, en comparación con aquellas que implican recompensas inmediatas con alto riesgo (Bechara et al., 1994; Petry, 2001b; Cavedini, Riboldi, Keller, D’ Annucci \& Bellodi, 2002; Manes et al., 2002), lo anterior se ha asociado a lesiones o afecciones en áreas como la corteza prefrontal ventromedial y la corteza dorsolateral (Blaszczynski \& Nower, 2002; Jameson, Hinson \& Whitney, 2004; Clark, Cools \& Robbins, 2004; Dretsch \& Tipples, 2008; Ochoa et al., 2013).

En este sentido, Goudriaan, Oosterlaan, de Beurs \& van den Brink (2006) sugieren una relación etiológica entre pacientes con trastornos adictivos no relacionados con sustancias y pacientes con trastornos adictivos por consumo de sustancias, en los cuales se evidencian importantes implicaciones, principalmente en la toma de decisiones; ya que el jugador patológico ignora constantemente las consecuencias negativas a largo plazo y centra su atención en la gratificación o recompensa inmediata sin considerar los riesgos que eso supone (Yechiam, Busemeyer, Stout, \& Bechara, 2005). Dicho déficit en la toma de decisiones se ha asociado a su vez con disfunción ejecutiva denotada en un deterioro de la flexibilidad cognitiva (Clark et al., 2004; Jameson et al., 2004; Dretsch \& Tipples, 2008), debido a alteraciones en circuitos frontales implicados en la toma de decisiones, lo cual refleja una génesis común entre jugadores patológicos y pacientes adictos a sustancias (Goudriaan, Oosterlaan, de Beurs \& van den Brink, 2006). 
De igual forma, se ha encontrado una estrecha correlación entre los déficits en la toma de decisiones y acciones impulsivas o desinhibidas del sujeto al momento de decidir, aunque no es imperativo que en todos los casos se presente dicha correspondencia (Busemeyer \& Stout, 2002; Van Holst et al., 2010). Por otra parte, y contrastando las nociones teóricas aportadas por el DSM-V respecto a la prevalencia del juego patológico, algunos estudios han reportado que, ante la toma de decisiones desventajosas, los hombres presentan mayores perseveraciones y/o conductas reiterativas ante situaciones o elecciones que conducen a perdidas, lo que no ocurre en las mujeres, en las cuales no se evidenciaron resultados significativos (Linnet et al., 2006).

\section{Medición y evaluación clínica}

Dentro de las técnicas de evaluación más implementadas en el juego patológico se encuentran las indirectas, como es el caso de la entrevista y los autoinformes, y las directas como la observación y registros psicofisiológicos. De estas, las ténicas más usadas son las primeras y dentro de ellas, las entrevistas; debido a que constituyen una herramienta fundamental para recolectar información del paciente en diferentes dimensiones, especialmente a nivel del historial clínico, conductas observables, manifestaciones del paciente, modo de interacción social y expectativas frente al tratamiento, etc., además que favorece el contacto inicial o rapport y con ello la relación terapeuta-paciente (Roa, 1995). Sin embargo, la entrevista no constituye el único instrumento de manejo en la intervención del juego patológico (Gómez et al., 2008; Gómez, 2015) pues a la par, son aplicados otros instrumentos complementarios que permiten recolectar los datos necesarios de una manera eficaz, amplia y completa.

Así, antes de la aplicación de cualquier técnica particular es importante realizar un diagnóstico previo de los aspectos o situaciones más relevantes a tratar según las manifestaciones del propio paciente, las cuales pueden contener implicaciones en el área personal, en cuyo caso debe abordar, por ejemplo, el grado de malestar producto de las conductas adictivas de juego, la presencial de patología dual o de tras- 
tornos comórbidos, las dificultades en la resolución de problemas y la toma de decisiones; o bien en las áreas familiar, social o laboral. A su vez, es importante conocer las expectativas que tiene el paciente frente a la terapia, esto es su deseo y voluntad de cambio, ya que de allí depende el éxito o no de la intervención terapéutica (Báez, Echeburúa \& Fernández-Montalvo, 1995). En el deseo de cambio, conviene implementar entrevistas de tipo motivacional, pues estas permiten que el sujeto sea participe de su proceso y se responsabilice del mismo, a la vez que es guiado paulatinamente por el profesional en tal tratamiento (Gómez et al., 2008).

Dentro de los instrumentos más implementados para evaluar concretamente el juego patológico, se encuentran la entrevista estructurada de la historia de juego (Echeburúa \& Báez, 1994), diferentes escalas de gravedad clínica como la Gambling Severity Index (Lesieur \& Blume, 1991), autoinformes como el 'Cuestionario de Juego Patológico de South Oaks’, SOGS (Echeburúa, Báez, Fernández-Montalvo \& Páez, 1994), el 'Cuestionario de juego de South Oaks-Versión Revisada para Adolescentes', SOGS-RA (Winters, Stinchfield \& Fulkerson,1993) y su versión en castellano (Secades \& Villa,1998), el 'Cuestionario de Evaluación de Variables Dependientes del Juego’ (Echeburúa \& Báez,1991), el 'Cuestionario de Juego de Massachussets', MAGS (Shaffer, Labrie, Scalan \& Cummings, 1994), el 'Inventario de Pensamientos sobre el Juego' (Echeburúa \& Báez,1991) y el 'Cuestionario Lie/Bet' (Johnson et al.,1997), entre otros.

\section{Abordaje e intervención clínica}

Los tratamientos más eficaces en el juego patológico han sido aquellos referentes a intervenciones farmacológicas y psicológicas, los cuales se han centrado especialmente en disminuir la cantidad de sesgos atencionales, y con ello el deseo y conductas impulsivas de juego (Franken, 2003). En general, se recomienda al jugador patológico realizar un tratamiento global que incluya ambas propuestas de intervención, con el objeto de conseguir mejores resultados. 


\section{Tratamiento farmacológico}

Cuando se habla de juego patológico es preciso considerar la influencia de tres neurotransmisores de especial importancia, la dopamina, la noradrenalina, y la serotonina, asociados respectivamente a la motivación y al sistema de recompensas, a la búsqueda de novedad y a la impulsividad (Hollander, Sood, Pallanti, Baldini-Rossi \& Baker, 2005). Al respecto de estos neurotransmisores, se han implementado una serie de fármacos en el juego patológico, como los antidepresivos ISRS o inhibidores selectivos de recaptación de serotonina, utilizados especialmente para la transmisión serotoninérgica (Bombín, 1992; Gómez et al., 2008), buscando con esto reducir los niveles de ansiedad e impulsividad, o bien, los síntomas asociados a trastornos comórbidos presentes en el jugador (Echeburúa \& Báez, 1990; Echeburúa, Salaberría \& Cruz-Sáez, 2014). Algunos de los antidepresivos más estudiados en esta patología son la fluoxetina, la sertralina, la paroxetina y el citalopram; aunque se han encontrado resultados divergentes entre estudio y estudio (Gómez et al., 2008; Gómez, 2015).

Otros tratamientos puestos a prueba en el juego patológico son los estabilizadores del estado de ánimo, que permiten controlar estados de ánimo negativos como la impulsividad (Gómez et al., 2008; Van den Brink, 2012; Potenza, 2012). Además, se han reportado resultados significativamente favorables frente al uso de antagonistas opioides que bloquean los receptores opioides y con ello la sensación de gratificación o euforia al momento de jugar, disminuyendo de este modo el pensamiento de juego, el ansia y con ello la dependencia o craving (Grant, Kim \& Hartman, 2008; Potenza, 2012; Gómez, 2015).

Como última medida, no se recomienda la utilización de ansiolíticos ya que estos pueden generar tolerancia y dependencia. En caso de que se requiera su uso, es importante suministrarse en el menor tiempo posible, específicamente en un periodo inferior a seis semanas (Gómez et al., 2008). No obstante, pese a la diversidad de tratamientos farmacológicos que se han probado en el juego patológico, aún no existe un medicamen- 
to que haya sido avalado científicamente para tratar específicamente este trastorno psiquiátrico (Grant, Odlaug \& Schreiber, 2012).

\section{Tratamiento psicológico}

Dada la clasificación del juego patológico como un trastorno de adicción, los tratamientos actualmente considerados se han centrado en intervenir la adicción en general, motivo por el cual esos tratamientos han sido probados en diversos trastornos de adicción por sustancias, como el abuso de alcohol (Becoña, 1996). Dentro de las técnicas implementadas se encuentran las terapias aversivas, la desensibilización imaginada o desensibilización sistemática sin orden de presentación de escenas, la relajación, el control de estímulos, la exposición en vivo con prevención de respuestas, la prevención de recaídas y la terapia cognitivo-conductual. Existe, además, otro tipo de intervención de gran relevancia como lo son las terapias grupales y los grupos de autoayuda, como jugadores anónimos, asociación de jugadores y juego controlado (Gómez et al., 2008; Gómez, 2015).

De las anteriores, las técnicas más abordadas y que mayor eficacia han demostrado son la exposición en vivo con prevención de respuestas, el control de estímulos y la desensibilización imaginada (Fernández-Montalvo \& Echeburúa, 1997). Otros autores incluyen a esta lista la terapia motivacional, la reestructuración cognitiva (Echeburúa, et al., 2014) y la prevención de recaídas (Gómez et al., 2008). Por su parte, Grant y Odlaug (2012), afirman que la terapia más conveniente en el juego patológico es la 'cognitivo-conductual', ya que la aplicación de las diferentes técnicas de esta terapia ha demostrado un porcentaje de efectividad del 50-80\% aproximadamente, con mayor duración de inhibición de la conducta a largo plazo (Grant \& Odlaug, 2012).

\section{Rehabilitación funcional del déficit frontal}

Tal como se ha abordado hasta aquí, las principales manifestaciones cognitivas, emocionales y comportamentales de los pacientes con trastorno por juego patológico, se vinculan estrechamente con el funcionamiento de los lóbulos frontales y las actividades ejecutivas pro- 
ducto de la actividad cerebral. En concordancia con lo anterior, en los últimos años se han implementado diferentes técnicas de rehabilitación, algunas de ellas dirigidas específicamente al tratamiento de las funciones ejecutivas (Levine et al., 2000; McDonald, Flashman, \& Saykin, 2002), esto debido a que pacientes con deterioro de dichas funciones, presentan diversos inconvenientes para gobernar su propia vida y responder a las demandas del entorno; especialmente si se trata de eventos imprevistos por la persona o de situaciones en las que es indispensable la atención, memoria, planeación, ejecución y toma de decisiones para lograr un resultado o meta (Muñoz-Céspedes \& Tirapu, 2001). Por tal razón, los objetivos de la rehabilitación de las funciones ejecutivas consisten en orientar o guiar la conducta de la persona en diferentes situaciones para que esta logre obtener unos resultados previamente fijados y el paciente pueda presentar mejoras en asuntos como la iniciación, regulación e inhibición del comportamiento, solución de problemas y autoconciencia de su enfermedad. Para ello, el rehabilitador neuropsicológico debe considerar aquellas áreas que se encuentran más preservadas, ya que le servirán de base para trabajar, posteriormente, sobre aquellas que presentan mayor alteración.

Debe conocerse, además, el grado de conciencia del paciente frente a su enfermedad y el nivel de afectación cognitiva, ya que de ello dependerá la eficacia de las técnicas a implementar (Arango \& Parra, 2008). En general se entrena al paciente en el aprendizaje de secuencias básicas de conductas cotidianas como, por ejemplo, vestirse y hacer las compras, o bien ejercicios más estructurados donde se requiera que la persona planifique y ejecute actividades orientadas a un fin particular. Sin embargo, la adecuación de una tarea u otra dependerá de las necesidades de cada paciente y de las estrategias que se utilicen en el tratamiento de los diferentes componentes de las funciones ejecutivas (Muñoz-Céspedes \& Tirapu, 2001).

En pacientes con implicaciones disejecutivas es común evidenciar problemas de espontaneidad, iniciación y secuenciación de la conducta, debido a compromisos importantes en los lóbulos frontales y en particular, en las áreas dorsolaterales, siendo esta dificultad más 
notoria para iniciar actividades o conductas relacionadas con variables externas más que con variables motivacionales internas y, cuando deciden realizar alguna actividad de manera voluntaria presentan complicaciones para seguir los pasos requeridos que los conducen al resultado esperado. En estos casos, la estrategia más implementada es enseñarles a los pacientes a relacionar varias conductas entre sí, de modo tal que al presentarse una de estas, la persona por asociación realice la otra instantáneamente (Arango y Parra, 2008).

En este sentido, un programa altamente utilizado actualmente para el tratamiento de problemas asociados a la iniciación y secuenciación de la conducta es el 'Everyday Activites to Sequence' (Daly \& Daly, 1996), en el cual se le muestra al paciente una serie de tarjetas que representan actividades de la vida cotidiana como lavar ropa, cepillarse los dientes o hacer compras; se elige una actividad particular, con sus respectivos pasos a seguir ilustrados en cuatro tarjetas y el neuropsicólogo le pide al paciente que identifique la actividad que se realiza y que organice las tarjetas de modo que tengan una secuencia lógica.

En lo correspondiente a la autorregulación de la conducta, uno de los programas más implementados es el de Meichenbaum \& Goodman (1971), quienes establecieron una serie de pasos con el objetivo final que los pacientes lograran interiorizar las instrucciones para la realización de alguna actividad. Para esto, el neuropsicólogo realiza inicialmente una actividad, dándose instrucciones verbales en presencia del paciente (modelo), luego le pide que realice la misma actividad mientras que él (el profesional) va mencionando en tono alto las instrucciones que debe seguir (guía externa explícita). Posteriormente, el rehabilitador le pide al paciente que repita la actividad pero que en esta ocasión sea él mismo quien mencione en tono alto las instrucciones (auto guía explícita). La cuarta fase consiste en que el paciente realice nuevamente la tarea repitiendo las instrucciones, esta vez en voz baja (auto guía) buscando, finalmente, que el paciente realice la actividad, teniendo conciencia de las instrucciones a seguir pero sin verbalizarlas (auto guía encubierta). 
De igual forma, para tratar problemas relacionados con la desinhibición de la conducta, se han utilizado especialmente las técnicas de modificación de la conducta, con el fin de disminuir las conductas inadecuadas y reforzar las adecuadas o positivas (Ponsford, 1995; Fernández, Rubin, Espinosa, Arango \& Pérez, 2002). Entre las más implementadas se encuentran la economía de dichas, la 'ténica de tiempo fuera', el 'modelado, el control de estímulos', y el 'reforzamiento positivo contingente'.

Como estrategia de entrenamiento en solución de problemas, a nivel clínico es utilizado el programa realizado por Von Cramon, Matthes-von Cramon \& Mai (1991) que tiene como propósito ayudar a los pacientes afrontar los problemas a través de la realización de diferentes pasos, entre ellos, identificar el problema, establecer objetivos, generar alternativas de solución, ejecutarlas y finalmente comprobarlas (Von Cramon et al., 1991).

En última instancia, para trabajar problemas de autoconciencia de la enfermedad, como lo señalan Arango \& Parra (2008) y Matter (1999), es habitual elaborar un proceso de psicoeducación con el paciente, brindándole información acerca de su patología y cómo esta tiene implicaciones en el modo de comportarse. También es importante darle a conocer en qué momentos está realizando una conducta inapropiada y por qué lo hace, se le pede al paciente que anote en una hoja la hora, fecha y frecuencia con la que realiza dicha conducta, con el objetivo de que la persona recuerde y preste mayor atención a ella. Otra estrategia pertinente es grabar al paciente en el momento en que realice dichas conductas para que él pueda verse y reconocer su comportamiento y la necesidad de modificarlo.

\section{Análisis Neurofuncional del Juego Patológico y sus correlatos biológicos}

Esta enfermedad, caracterizada por el desarrollo de conductas de juego patológico, tal como se ha profundizado hasta aquí desde diversos ángulos y disciplinas convergentes, ha sido abordada tradicionalmente por la psicología y la psiquiatría, siendo esta última la que en 
la mayoría de los casos es la única opción para el paciente con tratamientos farmacológicos, puesto que desde la psicología se cuenta con algunos avances en terapias de intervención clínica y que, sin embargo, pocos llegan a implementarse (por experticia o limitaciones de implementación) o a tener una eficacia real sobre los síntomas subyacentes a las conductas patológicas del funcionamiento cerebral. En este punto, es precisamente la actividad cerebral de este cuadro patológico sobre la que se debe profundizar antes de plantear un programa de intervención integral por parte de disciplinas médicas como Psiquiatría, Psicología y Neuropsicología. Lo anterior, conlleva a contemplar y estudiar ampliamente las vías neurales involucradas en cada una de las manifestaciones clínicas de la enfermedad, así como la interacción entre los circuitos neuronales y los procesos electroquímicos relacionados.

Dentro de los síntomas y compromisos relacionados con el juego patológico, se ha realizado ya aquí un recuento de las alteraciones del pensamiento, las emociones, la conducta y la cognición ante el desarrollo de la enfermedad, y sus correlatos anatómo-funcionales han orientado los estudios he investigaciones, dando cuenta de la existencia de desequilibrios de origen bioquímico de los lóbulos frontales y sus conexiones con regiones y estructuras límbicas en los pacientes.

En este sentido, el cuadro patológico más que abordarse como un trastorno mental debe estrictamente ser considerado como un 'Síndrome de Etiología Química' y de errores en la conectividad y sinapsis neuronal, Así debe ser asumido, como un síndrome prefrontal cuyos signos clínicos involucran a la manera de primeros síntomas manifestaciones psicológicas relacionadas con cuadros de ansiedad, agitación psicomotora, desarrollo de pensamientos y conductas desadaptativas que tienen como comorbilidad (no son desencadenantes) el desarrollo de deterioros funcionales de la actividad cerebral a nivel cognitivo, característicos en primera instancia por fallos en planeación, organización y manipulación de información, con compromisos en la memoria de trabajo y los procesos atencionales, llegando, según la severidad clínica de estos, a la posibilidad de progresos y dificultades que invo- 
lucren las funciones neuropsicológicas de forma general, más allá de actividades ejecutivas, aunque con diferencias en la intensidad de los compromisos patológicos.

Lo anterior, impactará necesariamente en las actividades de la vida diaria del paciente, afectando su handicap significativamente, repercutiendo en su estado emocional y limitando sus interacciones sociales. Los síntomas y signos deben estar presentes, como mínimo, en los últimos seis meses y sin ser consecuencia directa de daño cerebral adquirido (en cuyo caso se considerará como síndrome de juego patológico secundario ), de igual forma, no deben estar asociados a consumo de sustancias psicoactivas o síntomas de afecciones neurológicas congénitas que puedan servir para establecer un diagnóstico diferencial. Así, al considerar al juego patológico como un síndrome neuropsicológico de predomino frontal más que un trastorno meramente mental, el análisis y abordaje de sus síntomas y signos permitirá al clínico tener mayor flexibilidad al considerar la ocurrencia de estos, su intensidad y curso de desarrollo. Además, de contemplar la alta probabilidad de presentación, la comorbilidad con el síndrome obsesivo-compulsivo de prevalencia orbito-frontal.

Por tanto, el clínico tratante debe establecer para su diagnóstico la triada funcional de compromiso en funciones ejecutivas, pensamientos desadaptativos relacionados con el juego e implicaciones en la cotidianidad del sujeto, para así, formular dentro del programa integral de tratamiento neuropsicológico el abordaje de actividades orientadas a la rehabilitación cognitiva, el acompañamiento y manejo por psicología y psiquiatría, en pro de modificar los circuitos de recompensa/motivación y minimizar la búsqueda de conductas novedosas e impulsivas. Por su parte, psiquiatría deberá comprobar la presencia de desequilibrio en la segregación de dopamina, noradrenalina y/o serotonina, para establecer el tratamiento farmacológico ideal e individual para el paciente, más allá de pensarse en implementar la prescripción de medicamentos usuales a cualquier adicción o cuadro de ansiedad y depresión. 


\section{Conclusiones}

El juego patológico debe ser estudiado como un síndrome neuropsicológico de característica clínica prefrontal. A su vez, y partiendo de la nueva clasificación realizada por el DSM-5 respecto al juego patológico es importante formular y desarrollar nuevos estudios basados en estas últimas actualizaciones, donde se contemple el síndrome dentro de la categoría de las adicciones, ya que esto repercute en la manera como se aborda la patología (tanto en investigación, como en la intervención), desde su propia concepción, hasta la consideración de las manifestaciones clínicas, la etiología, los posibles modelos explicativos, la tipología, los criterios diagnósticos, las comorbilidades y los tratamientos propuestos con mayor eficacia.

Con respecto a la etiología es importante considerar, siempre que se vaya a realizar un diagnóstico, la multidimensionalidad del síndrome, especialmente por las nuevas modificaciones planteadas previamente y que suponen una mirada más global del mismo, en donde se consideren posibles como desencadenantes factores genéticos, bioquímicos, ambientales y sociales que probablemente estén asociados con patrones exclusivamente adictivos o particularmente con trastornos de adicción por sustancias.

De la tipología, es necesario distinguir al jugador patológico de otros tipos de jugadores, partiendo del nivel de gravedad o cronicidad de los síntomas. Al respecto, el DSM-5, presenta una clasificación general en cuanto a la intensidad y frecuencia de los síntomas, pero dicha tipificación puede variar de acuerdo al marco de referencia o al autor que se tome. La clasificación que se haga puede igualmente servir de base al momento de considerar los criterios diagnósticos que cumple o que se evidencian en el sujeto.

Otro aspecto importante al momento de realizar el diagnóstico de juego patológico es la comorbilidad o presencia de patología dual, por lo que es clave efectuar un diagnóstico diferencial que le permita al profesional de la salud distinguir claramente entre el síndrome contemplado y otros trastornos de adicción, por ejemplo. Aquí se hace im- 
perativo recordar las características propias del juego patológico que se basan en la persistencia, recurrencia e incontrolabilidad de las conductas respecto al juego y alteraciones cognitivas de tipo ejecutivo, así como una sensación displacentera en el sujeto e implicaciones graves en sus dimensiones social, laboral, familiar y personal. Así mismo, es importante considerar el tiempo de duración o presentación de los síntomas, que debe ser de al menos seis meses, pese a que se plantee que sean 12 según los criterios establecidos en el DSM-5.

Con relación a los tratamientos propuestos es ineludible aclarar que el mero tratamiento farmacológico o psicológico por separado no tendrá tanto efecto en el paciente, como si se emplean ambos en el proceso de intervención. De igual forma, el profesional debe elegir cuál tratamiento es el más conveniente y cuál tendrá mayor efecto a largo plazo, ya que no se obtendrán los mismos resultados en todos los pacientes, esto dependerá de muchos factores como la presencia de trastornos comórbidos tales como la depresión y la bipolaridad y variables sociodemográficas, entre ellas, la edad, el género y el tiempo de juego.

En la intervención se debe contemplar que a la fecha no existen ni medicamentos ni terapias centradas exclusivamente en el juego patológico y, además, son pocas las personas que acuden a tratamiento, siendo menor el porcentaje de hombres que de mujeres, y entre aquellos que deciden iniciarlo son pocos los que terminan el proceso con éxito, por lo cual, el tratamiento puede verse gravemente impedido u obstaculizado. Para esto, es preciso intervenir con un programa que oriente a la rehabilitación neuropsicológica, donde, dando prioridad al funcionamiento cognitivo y el impacto que su deterioro traerá a la vida diaria del paciente, se vincule la motivación para no decaer funcionalmente, más que insistir en la presencia de enfermedades mentales y conductas anormales que lo que logran es aislar al sujeto de la atención clínica.

En síntesis, es evidente que el juego patológico representa un cuadro complejo, socialmente hablando, esto debido a la legalización de establecimientos que promueven los juegos de azar, por un lado, y por el otro, a un desconocimiento general del síndrome y sus correlatos 
neurobiológicos, fruto de la poca exploración en este campo, con los pocos estudios realizados desde la psicología y, aún menos, desde la neuropsicología. Tal como se mencionó en apartados iniciales, son escasos los estudios en torno a alteraciones neuropsicológicas, los pocos hallados en la literatura, son investigaciones internacionales que se han centrado en estudiar exclusivamente la función ejecutiva dejando de lado la consideración de otras variables y componentes cognitivos de importancia; sin embargo, si se compara con Colombia, son exiguas las investigaciones neuropsicológicas y prácticamente nulas las propuestas de rehabilitación neuropsicológica para esta patología, de allí la importancia de plantear y ejecutar nuevos estudios que ahonden en este campo tan poco explorado actualmente. 


\section{Referencias}

American Psychiatric Association [APA]. (2014). Diagnostic and statistical manual of mental disorders (DSM-5), 5th Edition. Arlington, VA: American Psychiatric Publishing.

Arango, J.C., Parra, M.A. (2008). Rehabilitación de las Funciones Ejecutivas en caso de patología cerebral. Revista Neuropsicología, Neuropsiquiatría y Neurociencias, 8(1), 159-178.

Báez, C., Echeburúa, E., Fernández-Montalvo, J. (1995). Variables predictoras de abandonos y recaídas en el juego patológico. Revista Análisis y modificación de conducta, 21(75):5-22

Bahamon, M. (2006). Juego patológico. Revisión de tema. Revista Colombiana de Psiquiatría, 35(3), 380-399.

Barry, D., Petry, N.M. (2008). Predictors of decisión-making on the lowa Gambling Task: independent effects of lifetime history of substance use disorders and performance on the Trail Making Test. Brain and Cognition, 66(3), 243-252. Doi: 10.1016/j.bandc.2007.09.001

Bechara, A., Damasio, A.R., Damasio, H., Anderson, S.W. (1994). Insensitivity to future consequences following damage to human prefrontal cortex. Cognition, 5O(1-3), 7-15. Doi: 10.1016/0010-0277(94)90018-3

Becoña, E. (1996). Tratamiento del juego patológico. En:. Tratamiento psicológico de hábitos y enfermedades. Madrid: Pirámide.

Blaszczynski, A., Nower, L. A. (2002). A pathways model of problem and pathological gambling. Addict Abingdon Engl, 97(5): 487-99.

Bombín, B. (1992). Enfoques farmacológicos: serotonina y ludopatía. En: Bombín, B. (ed): El juego de azar: patología y testimonios. Valladolid: Junta Castilla y León. Busemeyer, J.R., Stout, J.C. (2002). A contribution of cognitive decisión models to clinical assessment: decomposing performance on the Bechara gambling task. Psychological Assessment, 14(3), 253-262. 
Butcha, R.M. (1995). Gambling among adolescents. Clin Pediatr (Phila), 34(7), 346-348. Cavedini, P., Riboldi, G., Keller, R., D’Annucci, A., Bellodi, L. (2002). Frontal lobe dysfunction in pathological gambling patients. Biological Psychiatry, 51(4), 334-341.

Clark, L., Cools, R., Robbins, T.W. (2004). The neuropsychology of ventral prefrontal cortex: decisión-making and reversal learning. Brain and Cognition, 55(1), 41-53. Doi: 10.1016/So278-2626(03)00284-7.

Comisión Nacional del Juego (2001). Memoria 20oo. Madrid: Ministerio de Justicia e Interior: Comisión Nacional del Juego.

Daly, M. P., Daly, D. P. (1996). Everyday Activities to Sequence. Austin, TX: PROED, Inc.

Dretsch, M.N., Tipples, J. (2008). Working memory involved in predicting future outcomes base don past experiences. Brain and cognition, 66(1), 83-90. Doi: 10.1016/j.bandc.2007.05.006

Echeburúa, E. (2006). Avances en el tratamiento psicológico de la ludopatía y de las nuevas adicciones. Revista de Formación Continuada en Trastornos Adictivos de la Sociedad Española de Toxicomanías, 2: 169-186.

Echeburúa, E., Báez, C. (1990). Enfoques terapéuticos en el tratamiento psicológico del juego patológico. Revista Española de Terapia del Comportamiento, $8(2), 127-146$.

Echeburúa, E., Báez, C., Fernández-Montalvo, J., Páez, D. (1994). Cuestionario de Juego Patológico de South Oaks (SOGS): Validación española. Análisis y Modificación de conducta, 20 (74), 769-791.

Echeburúa, E., Báez, C. (1994). Concepto y evaluación del juego patológico. En: Graña J. (ed). Conductas adictivas. Madrid: Pirámide.

Echeburúa, E., Salaberría, K., Cruz-Sáez, M. (2014). New Challenges in the Treatment of Disordered Gambling. Revista Terapia Psicológica. 32(1); 31-40. Doi: 10.4067/So718-48082014000100003. 
Fernández, S., Rubin, E., Espinosa, A., Arango, J., Pérez, E. (2002). Manejo de las conductas de agresividad en pacientes con probable enfermedad de Alzheimer. En Reunión sobre Daño Cerebral y Calidad de Vida (ed. $7^{\circ}$, 2002). Cerebro y Salud Mental, (pp. 368-382). Madrid, España: MAPFRE, S. A.

Fernández-Montalvo, J., Echeburúa, E. (1997). Manual práctico del juego patológico. Madrid: Pirámide.

Forbush, K.T., Shaw, M. Graeber, M.A. Hovick, L., Meyer, V.J., Moser, D.J., Bayless, J., ... Black, D.W. (2008). Neuropsychological characteristics and personality traits in pathological gambling. CNS Spectrums, 13(4), 306-315. Doi: 10.1017/ S1092852900016424

Franken, I.H.A. (2003). Drug craving and addiction: integrating psychological and neuropsychopharmacological approaches. Prog Neuropsychopharmacol Biol Psychiatry, 27(4):563-79.

Gómez, J., Sancho, M., Fernández, M.M., Cabrera, J., Quesada, C., Berrocoso, J., Martín, J.C. (2008). Evaluación del juego patológico. En: Santos Cansado, J. (coord). Manual de intervención en juego patológico.

Gómez, M. (2006). Esquizofrenia y Juego Patológico. Revista de Formación Continuada en Trastornos Adictivos de la Sociedad Española de Toxicomanías, 2, 87-103.

Gómez, M. (2015). Clinical implications of schizophrenia in pathological gambling. Revista de Patología Dual. 2(4):22; 1-10. Doi: 10.17579/RevPatDual.02.22

González, A., Aymami, M.N., Jiménez, S., Doménech, J.M., Granero, R. y LouridoFerreira, M.R. (2003). Assessment of pathological gamblers who use slot machines. Psychological Reports, 93, 707-716. Doi: 10.2466/pro.2003.93.3.707

Goudriaan, A.E., Oosterlaan, J., de Beurs, E., Van den Brink, W. (2006). Neurocognitive functions in pathological gambling: a comparison with alcohol dependence, Tourette syndrome and normal controls. Addict Abingdon Engl, 101(4): 534-47. 
Grant, J. E., Odlaug, B. L., Schreiber, L. N. (2012). Pharmacological Treatments in Pathological Gambling. British Journal of Clinical Pharmacology, 77(2), 375831. Doi: 10.111/j.1365-2125.2012.04457.x

Grant, J. E., Odlaug, B. L. (2012). Psychosocial Interventions for Gambling Disorders. In: National Center for Responsible Gaming (NCRG). (2012). Increasing the odds (pp. 38-51). Boston.

Grant, J.E., Kim, S.W., Hartman, B.K. (2008). A double-blind, placebo-controlled study of the opiate antagonist naltrexone in the treatment of pathological gambling urges. $J$ Clin Psychiatry, 69(5):783-9.

Hollander, E., Sood, E., Pallanti, S., Baldini-Rossi, N. Baker, B. (2005). Pharmacological treatments of pathological gambling. Journal Gambling Studies. 21(1): 99-110. Doi: 10.1007/s10899-004-1932-8

Jameson, T.L., Hinson, J.M., Whitney, P. (2004). Components of working memory and somatic markers in decisión making. Psychonomic bulletin review, 11(3), 515-520.

Johnson, E.E., Hamer, R., Nora, R.M., Tan, B., Eisentsein, N., Engerhart, C. (1997). The Lie/Bet questionnaire for screening pathological gamblers. Psychological Reports, 8o(1):83-88.

Leiserson, V., Pihl, R.O. (2007). Reward-sensitivity, inhibition of reward-seeking, and dorsolateral prefrontal working memory function in problem gamblers not in treatment. Journal of gambling studies cosponsored by the National Council on Problem Gambling and Institute for the study of Gambling and Commercial Gaming, 23(4), 435-455. Doi: 10.1007/s10899-007-9065-5

Lesieur, H.R., Blume, S.B. (1991). Evaluation of patients treated for pathological gambling in a combined alcohol, substance abuse and pathological gambling treatment unit using the addiction severety index. Br J Addict, 86(8):1017-1028.

Lesieur, H.R., Rosenthal, R.J.(1991). Pathological gambling: A review of the literature. (prepared for the American Psychiatric Association task force on DSM-IV 
committee on disorders of impulse control not elsewhere classified). $J$ Gambl Stud,7(1):5-39. Doi: 10.1007/BFo1019763.

Levine, B., Robertson, I. H., Clare, L., Carter, G., Hong, J., Wilson, B. A., Duncan, J., Stuss, D.T. (2000). Rehabilitation of executive functioning: An experimental-clinical validation of goal management training. Journal of the International Neuropsychological Society, 6(3), 299-312. Doi: 10.3389/fnhum.2011.00009

Linnet, J., Rojskjaer, S., Nygaard, J., Maher, B.A. (2006). Episodic chasing in pathological gamblers using the lowa gambling task. Scandinavian Journal of Psychology, 47(1), 43-49. Doi: 10.1111/j.1467-9450.2006.00491.x

Manes, F., Sahakian, B., Clark, L., Rogers, R., Antoun, N., Aitken, M., Robbins, T. (2002). Decision-making processes following damage to the prefrontal cortex. Brain. A journal of neurology, 125(3), 624-639. Doi: 10.1093/brain/awfo49.

Marazziti, D., Catena, M., Osso, D., Conversano, C., Consoli, G., Vivarelli, L., Mungai, F., Golia, F. (2008). Clinical practice and epidemiology executive function abnormalities in pathological gamblers. Clinical practice and Epidemiology in Mental Health, 6, 1-6. Doi: 10.1186/1745-0179-4-7

Mateer, C. A. (1999). The rehabilitation of executive disorders. En D. T. Stuss, G. Winocur, \& I. Robertson (Eds.), Cognitive neurorehabilitation, (pp. 314-332). Cambridge, England: Cambridge University Press.

McDonald, B. C., Flashman, L. A., Saykin, A. J. (2002). Executive dysfunction following traumatic brain injury: Neural substrates and treatment strategies. NeuroRehabilitation, 17(4), 333-344.

Meichenbaum, D. H., Goodman, J. (1971). Training impulsive children to talk to themselves: A means of developing selfcontrol. Journal of Abnormal Psychology, 77(2), 115-126. Doi: 10.1037/hoo30773.

Monaghan, S., Blaszczynski , A., Nower, L. (2009). Consequences of winning: the role of gambling outcomes in the development of irrational beliefs. 
Behavioural and Cognitive Psychotherapy, 37(1), 49-59. Doi: 10.1017/ S135246580800502X.

Muñoz-Céspedes, J.M., Tirapu, J. (2001). Rehabilitación neuropsicológica. Madrid: Síntesis.

Ochoa, C., Álvarez-Moya, E.M., Penelo, E., Aymami, M.N., Gómez-Peña, M., Fernández-Aranda, F.,...Jiménez-Murcia, S. (2013). Decision-making deficits in pathological gambling: the role of executive functions, explicit knowledge and impulsivity in relation to decisions made under ambiguity and risk. The American Journal on Addictions, 22(5):492-4999. Doi: 10.1111/j.15210391.2013.12061.x

Ochoa, C., Álvarez-Moya, E.M., Penelo, E., Aymami, M.N., Gómez-Peña, M., Ochoa, E., Labrador, F.J. (1994). Juego patológico. Barcelona: Plaza y Janés.

Organización Mundial de la Salud [OMS]. (1994). Clasificación Internacional de Las Enfermedades. Trastornos Mentales y del Comportamiento: Descripciones Clínicas y pautas para el Diagnóstico (CIE-1O), 10 a revisión. Madrid: Panamericana.

Petry, N.M. (2001a). Pathological gamblers, with and without substance use disorders, discount delayed reward at high rates. Journal of Abnormal Psychology, 11O(3),482-487. Doi: 10.1037/0021-843X.110.3.482

Petry, N.M. (2001b).Substance abuse, pathological gambling, and impulsiveness. Drug and Alcohol Dependence, 63(1), 29-38. Doi: 10.1016/So3768716(00)00188-5

Ponsford, J. (1995). Assessment and management of behaviour problems associated with TBI. En S. Sloan \& P. Snow (Eds.), Traumatic brain injury: Rehabilitation for everyday adaptive living. East Sussex, UK: Lawrence Erlbaum Associates.

Potenza, M. N. (2012). Pharmacological Approaches to Treating Pathological Gambling. In: National Center for Responsible Gaming (NCRG). (2012). What 
Clinicians Need to Know About Gambling Disorders. Increasing the Odds. (vol 7, pp. 52-60): Washington DC.

Prieto, M. (2003). Una propuesta de clasificación de los jugadores patológicos. Psicología Conductual, 11(1), 5-23.

Roa, A. (1995). Evaluación en psicología clínica y de la salud. Madrid: CEPE.

Rugle, L., Melamed, L. (1993). Neuropsychological assessment of attention problems in pathological gamblers. Journal of Nervous Mental Disease, 181(2), 107-112.

Secades, R., Villa, A. (1998). El juego patológico; Prevención, evaluación y tratamiento en la adolescencia. Madrid: Pirámide.

Shaffer, H.J., LaBrie, R., Scanlan, K.M., Cummings, T.N. (1994). Pathological gambling among adolescents: Massachusetts Gambling Screen (MAGS). Journal of Gambling Studies, 10(4), 339-362. Doi: 10.1007/BFo2104901.

Unwin, B.K., Davis, M.K., De Leeuw, J.B. (2000). Pathologic gambling. Am Fam Physician, 61(3):741-749.

Van den Brink, W. (2012). Evidence-based pharmacological treatment of substance use disorders and pathological gambling. Curr Drug Abuse Rev, 5(1):3-31. Doi: 10.2174/1874473711205010003

Van Holst, R.J., Van Den Brink, W., Veltman, D.J., Goudriaan, A.E. (2010). Neuroscience and Biobehavioral Reviews Why gamblers fail to win: A review of cognitive and neuroimaging findings in pathological gambling. Neuroscience Biobehavioral Reviews, 34(1),87-107. Doi: 10.1016/j.neubiorev.2009.07.007.

Verdura, E.J. (2014). Juego patológico, adicción sin sustancia. (Tesis de doctorado). Universidad Computlense de Madrid, Madrid, España.]

Volberg, R.A., Abbott, M.W. (1994). Lifetime prevalence estimates of pathological gambling in New Zealand. Int J Epidemiol, 23(5),976-998.

Von Cramon, D. Y., Matthes-von Cramon, G., Mai, N. (1991). Problem-solving deficits in brain-injured patients: A therapeutic approach. Neuropsychological Rehabilitation, 1(1), 45-64. Doi: 10.1080/09602019108401379. 
Winters, K.C., Stinchfield, R.D., Fulkerson, J. (1993). Toward the development of an adolescent gambling problem severity scale. Jornal of Gambling Studies, 9(1), 63-84. Doi: 10.1007/BF01019925

Yechiam, E., Busemeyer, J.R., Stout, J.C., Bechara, A. (2005). Using cognitive models to map relations between neuropsychological disorders and human decisión-making déficits. Psychological Science, 16(12), 973-978.

Zeitlin, H. (1994). Children with alcohol misusing parents. Br Med Bull, 5O(1),139-51.

Zuluaga, P.A., Montoya, L.F. (2005). Características de la Función Ejecutiva en personas con cuadro clínico de Juego Patológico: Un estudio descriptivo comparativo con jugadores patológicos del área metropolitana del Valle de Aburrá. Poiésis, 9, 1-4, 